\title{
Role of thrombophilic factors in full-term infants with neonatal encephalopathy
}

\author{
Johanna C Harteman' ${ }^{1}$, Floris Groenendaal' ${ }^{1}$, Manon JNL Benders' ${ }^{1}$ Albert Huisman², Henk J Blom ${ }^{3}$ and Linda S de Vries ${ }^{1}$
}

BACKGROUND: Neonatal encephalopathy (NE) is a serious condition, primarily seen following hypoxia-ischemia (HI). Two different patterns of brain injury can be recognized on magnetic resonance imaging (MRI): white matter/watershed (WM/WS) or basal ganglia/thalamus (BGT) injury. Whether these patterns of injury can be attributed to different associated risk factors still needs to be established.

METHODS: In 118 infants with clinical signs of NE following perinatal $\mathrm{HI}$, thrombophilic factors, such as factor $\mathrm{V}$ Leiden and prothrombin gene mutation, C677T and A1298C polymorphisms in the methylenetetrahydrofolate reductase (MTHFR) gene, and plasma levels of homocysteine and lipoprotein(a), were prospectively investigated. Antenatal and perinatal variables were studied.

RESULTS: WM/WS injury was seen in 45 infants, BGT injury in 40, and normal neuroimaging in 33. Antenatal factors did not differ across these groups. The BGT pattern was associated with lower Apgar scores, whereas the WM/WS pattern was associated with hypoglycemia $(<2.0 \mathrm{mmol} / \mathrm{l})$, CT or TT 677 polymorphism in the MTHFR gene, and plasma homocysteine levels in the upper quartile.

CONCLUSION: In infants with NE following perinatal Hl, the WM/WS pattern of injury was associated with hypoglycemia, the MTHFR 677CT or TT genotype, and higher levels of plasma homocysteine. BGT injury showed an association with signs suggestive of acute $\mathrm{HI}$.

N eonatal encephalopathy (NE) is a serious condition occurring in 1-2 of every 1,000 live term births (1). Although $\mathrm{NE}$ is a heterogeneous disorder, it is mostly encountered following perinatal hypoxia-ischemia (HI) (2). Using magnetic resonance imaging (MRI), two main patterns of injury can be distinguished, predominantly involving the basal ganglia-thalamus (BGT) or the white matter/watershed region (WM/WS). The pattern of injury depends mainly on the severity and duration of the HI insult $(3,4)$. BGT injury is often preceded by a sentinel event, whereas the WM/WS pattern is commonly seen in the context of long-standing antenatal risk factors $(3,5)$.

However, neonates with similar degrees of HI may differ in the extent of brain injury, ranging from none to near total brain injury (6). It is unclear whether these patterns in neonates can be attributed to different antenatal or perinatal risk factors. Miller et al. (7) showed similar antenatal and perinatal conditions across infants with NE and BGT, WM/WS injury, or normal neuroimaging (7). In a study by Mercuri et al. (6), $28 \%$ of neonates presenting with NE with Apgar scores $\leq 3$ had normal MRI scans or minimal white matter changes, whereas $95 \%$ of the infants with Apgar scores $>7$, but abnormalities on clinical assessment within $48 \mathrm{~h}$ after birth, had abnormal scans. This might suggest that other factors, in particular genetic factors, predispose to HI-induced perinatal brain injury.

The pathogenesis of brain damage after $\mathrm{HI}$ is therefore complex. Recent research has focused on several mechanisms, including excitotoxicity, oxidative stress, inflammation, and coagulation. There has been increasing interest in the contribution of individual (genetic) factors interfering with these mechanisms. These may explain differences in the underlying vulnerabilities to preterm and full-term neonatal brain injury (8). Previous studies have focused on genes related to inflammatory or coagulation pathways (9).

The aim of this study was to determine whether there is an association between perinatal and thrombophilic risk factors and the predominant type of brain injury in full-term infants with NE following HI. On the basis of the previous literature, we hypothesized that BGT lesions are associated with profound HI (10). However, we expected that more (thrombophilic) risk factors are present in infants with WM/WS injury as compared with those with normal neuroimaging.

\section{RESULTS}

A total of 118 infants with a median gestational age of 40 $3 / 7$ wk (range 36 2/7-43 2/7) were enrolled in the study. A neonatal MRI was performed in 88 infants (75\%) with a median age of $5 \mathrm{~d}$ (range 2-15d). Fourteen infants underwent serial cranial ultrasonography (cUS). MRI was not performed in the absence of neonatal seizures and cUS abnormalities or in the presence of only small periventricular densities $(n=2)$. The other 16 infants died before an MRI could be performed, and postmortem examination was available in 6 infants.

\footnotetext{
'Department of Neonatology, Wilhelmina Children's Hospital, Utrecht, The Netherlands; ${ }^{2}$ Department of Hematology and Clinical Chemistry, University Medical Center, Utrecht, The Netherlands; ${ }^{3}$ Department of Clinical Chemistry, Metabolic Unit, Free University (VU) Medical Center, Amsterdam, The Netherlands.

Correspondence: Linda S. de Vries (I.s.devries@umcutrecht.nl)
} 


\section{Neuroimaging}

On the basis of MRI or cUS, 45 infants (38\%) had a predominant WM/WS pattern of injury, 40 infants (34\%) had predominant BGT lesions, and in 33 infants (28\%) cUS and/or MRI did not reveal any abnormalities (Table 1). Because of severe cUS findings and a flat trace on electroencephalogram (EEG) indicating a poor outcome, MRI was performed early (day 2) in eight infants before withdrawal of intensive care was considered.

\section{Clinical Data}

Table 1 presents antenatal, perinatal, and postnatal factors across the three groups. There were significant differences with regard to emergency cesarean section (emCS), ventouse delivery, umbilical pH, Apgar score, hypoglycemia $<2.0 \mathrm{mmol} / \mathrm{l}$, and therapeutic hypothermia. The group with predominant BGT injury had more severe acidosis, lower Apgar scores, and the highest percentage of emCS. Hypoglycemia $<2.0 \mathrm{mmol} / \mathrm{l}$ was most frequent in the WM/WS group. Hypothermia was more often applied in the groups of infants without brain injury and with BGT injury. Infants without brain injury had less often seizures as compared with those in the WM/WS and BGT groups.

\section{Thrombophilic Factors}

Results of screening of the thrombophilic factors are presented in Table 2. Blood was drawn at a median age of $2 \mathrm{~d}$ after birth (range 0-14 d). Investigation of activated protein $\mathrm{C}$ resistance and/or genotyping of factor $\mathrm{V}$ Leiden mutation was successfully performed in $100 \%$ of the infants, genotyping of the prothrombin gene mutation in $92 \%$, and methylenetetrahydrofolate reductase (MTHFR) polymorphisms in $100 \%$. Plasma lipoprotein(a) (Lp(a)) levels were measured in 103/118 infants and total homocysteine (tHcy) in 102/118. Lp(a) ranged from $<13$ to $206 \mathrm{mg} / \mathrm{l}$. In $43 / 103$ (42\%) samples, Lp(a) level was $<13 \mathrm{mg} / \mathrm{l}$. As compared with infants without brain injury, infants with WM/WS had a significantly higher prevalence of heterozygous 677CT and homozygous 677TT MTHFR genotype (Table 3 ). tHcy across the three groups ranged between 0 and $14.0 \mu \mathrm{mol} / \mathrm{l}$. The mean level of tHcy differed significantly $(P=0.001)$ across the no brain injury, WM/WS, and BGT groups $(5.43,6.67$, and $4.24 \mu \mathrm{mol} / \mathrm{l}$, respectively). In the WM/WS group, significantly more infants had a tHcy in the highest quartile as compared with the no brain injury group (Figure 1). Table 4 shows the lack of association of MTHFR genotype and tHcy.

\section{Multinomial Logistic Regression Analysis}

The full multinomial model included sex, hypothermia, hypoglycemia $(<2.0 \mathrm{mmol} / \mathrm{l})$, Apgar score $<5$ after $5 \mathrm{~min}$, and MTHFR 677 CT or TT genotype. Variables that were significant in the univariable analysis, such as emCS, ventouse delivery, umbilical $\mathrm{pH}$, seizures, and death, were not included

Table 1. Univariate risk factors for WM/WS or BGT pattern of injury following NE in term and near-term infants ( $n$ (\%) or median (range))

\begin{tabular}{|c|c|c|c|c|}
\hline Characteristic & Normal $(n=33)$ & WM/WS $(n=45)$ & $\mathrm{BGT}(n=40)$ & $P$ value \\
\hline Male/female & $20 / 13$ & $26 / 19$ & $23 / 17$ & 0.96 \\
\hline Gestational age (wk) & $41.0(37.3-43.3)$ & $40.4(36.3-42.0)$ & $40.1(37.1-42.1)$ & 0.09 \\
\hline Preeclampsia & $1(3.0)$ & $4(8.9)$ & $5(12.5)$ & 0.35 \\
\hline PROM & $1(3.0)$ & $1(2.2)$ & $1(2.5)$ & 0.98 \\
\hline Maternal infection & $4(12.1)$ & $4(8.9)$ & $1(2.5)$ & 0.28 \\
\hline Fetal bradycardia & $28 / 31(90.3)$ & $33 / 40(82.5)$ & $28 / 35(80.0)$ & 0.50 \\
\hline Meconium-stained liquor & $20 / 30(67.0)$ & $27 / 38(71.1)$ & $17 / 27(63.0)$ & 0.78 \\
\hline Ventouse delivery & $10(30.3)$ & $9(20.5)$ & $3(7.5)$ & 0.04 \\
\hline Emergency cesarean section & $10(30.3)$ & $22(48.9)$ & $25(62.5)$ & 0.02 \\
\hline \multicolumn{5}{|l|}{ Postpartum } \\
\hline Birth weight $<5$ th percentile & $2(6.1)$ & $4(8.9)$ & $2(5.0)$ & 0.76 \\
\hline Head circumference $(\mathrm{cm})$ & $35.0(32.0-40.0)$ & $35.0(32.0-38.5)$ & $34.0(33.0-38.0)$ & 0.53 \\
\hline Umbilical artery $\mathrm{pH}$ & $6.98(6.62-7.30)$ & $7.02(6.62-7.28)$ & $6.91(6.40-7.29)$ & 0.03 \\
\hline Neonatal seizures & $16(48.5)$ & $34(75.6)$ & $34(85.0)$ & 0.00 \\
\hline Multiorgan failure & $23 / 29(79.3)$ & $36 / 39(92.3)$ & $37 / 40(92.5)$ & 0.16 \\
\hline Hypoglycemia $<2.0 \mathrm{mmol} / \mathrm{l}$ & $4(12.1)$ & $19(42.2)$ & $8(20.0)$ & 0.01 \\
\hline Therapeutic hypothermia & $18(54.5)$ & $13(28.9)$ & $25(62.5)$ & 0.01 \\
\hline Death & 0 & $10(22.2)$ & $28(70.0)$ & 0.00 \\
\hline
\end{tabular}

Values in bold, $P<0.05$.

$B G T$, basal ganglia-thalamus; NE, neonatal encephalopathy; PROM, prolonged rupture of membranes; WM/WS, white matter/watershed region. 
Table 2. Thrombophilic investigations of term infants with NE after perinatal asphyxia $(n, \%)$

\begin{tabular}{|c|c|c|c|c|}
\hline & $\begin{array}{l}\text { Normal } \\
(n=33)\end{array}$ & $\begin{array}{l}\text { WM/WS } \\
(n=45)\end{array}$ & $\begin{array}{c}\text { BGT } \\
(n=40)\end{array}$ & $\begin{array}{c}P \\
\text { value }\end{array}$ \\
\hline Factor V Leiden & 0 & 3 & 0 & 0.08 \\
\hline Prothrombin gene & 0 & 2 & 2 & 0.43 \\
\hline APCR (mean, range) & $\begin{array}{c}1.0 \\
(0.82-1.13)\end{array}$ & $\begin{array}{c}0.98 \\
(0.67-1.14)\end{array}$ & $\begin{array}{c}0.97 \\
(0.75-1.12)\end{array}$ & 0.25 \\
\hline $\mathrm{APCR}<0.94^{\mathrm{a}}$ & 7 & 13 & 6 & 0.36 \\
\hline Lupus anticoagulans & 0 & 0 & 0 & NA \\
\hline $\begin{array}{l}\text { Antiphospholipid } \\
\text { antibodies }^{\mathrm{b}}\end{array}$ & 0 & 0 & 0 & NA \\
\hline $\begin{array}{l}\text { Lipoprotein(a) } \\
(\mathrm{mmol} / \mathrm{l})(\text { mean, } \\
\text { range) }\end{array}$ & $\begin{array}{c}41.0 \\
(13-144)\end{array}$ & $\begin{array}{c}46.2 \\
(13-206)\end{array}$ & $\begin{array}{c}34.6 \\
(13-141)\end{array}$ & 0.65 \\
\hline $\begin{array}{l}\text { Homocysteine } \\
>6.4 \mu \mathrm{mol} /\left.\right|^{c}\end{array}$ & $7 / 32$ & $18 / 38$ & $3 / 32$ & 0.00 \\
\hline
\end{tabular}

Values in bold, $P<0.05$

APCR, activated protein C resistance; BGT, basal ganglia-thalamus; Ig, immunoglobulin; NA, not available; NE, neonatal encephalopathy; WM/WS, white matter/watershed region ${ }^{a}$ No brain injury group is reference, below 25 th percentile. ${ }^{\mathrm{C} C o m p r i s e s}$ anticardiolipin IgG and IgM and anti- $\beta$-2-glycoprotein-1 lgG and lgM antibodies. "No brain injury group is reference, above 75 th percentile.

Table 3. MTHFR 677 and 1298 polymorphisms

\begin{tabular}{lccccc}
\hline Genotype & $\begin{array}{r}\text { Normal } \\
(n=33)\end{array}$ & $\begin{array}{c}\text { WM/WS } \\
(n=45)\end{array}$ & $\begin{array}{c}\text { OR }(95 \% \mathrm{Cl}) \\
\text { WM/WS vs. } \\
\text { Normal }\end{array}$ & $\begin{array}{c}\text { BGT } \\
(n=40)\end{array}$ & $\begin{array}{c}\text { OR }(95 \% \mathrm{Cl}) \\
\text { BGT vs. Normal }\end{array}$ \\
\hline MTHFR 677 & & & & & \\
CC & $24(73)$ & $17(38)$ & $1^{\mathrm{a}}$ & $19(48)$ & $1^{\mathrm{a}}$ \\
CT & $8(24)$ & $21(47)$ & $3.7(1.3-10.3)^{*}$ & $18(45)$ & $2.8(1.0-7.9)$ \\
TT & $1(3)$ & $7(16)$ & $9.9(1.1-87.9)^{*}$ & $3(8)$ & $3.8(0.4-39.4)$ \\
MTHFR 1298 & & & & & \\
AA & $10(30)$ & $17(38)$ & $1^{\mathrm{a}}$ & $21(53)$ & $1^{\mathrm{a}}$ \\
AC & $17(52)$ & $23(51)$ & $0.8(0.3-2.2)$ & $17(43)$ & $0.2(0.03-0.94)$ \\
CC & $6(18)$ & $5(11)$ & $0.5(0.1-2.0)$ & $2(5)$ & $0.2(0.03-1.2)$ \\
\hline
\end{tabular}

$\mathrm{BGT}$, basal ganglia-thalamus; $\mathrm{Cl}$, confidence interval; MTHFR, methylenetetrahydrofolate reductase; OR, odds ratio; WM/WS, white matter/watershed region.

aReference category.

${ }^{*} P<0.05$.

to avoid multicollinearity. The model was reduced based on Akaike's information criterion. Interactions could not be demonstrated. Multinomial logistic regression analysis showed the lowest Akaike's information criterion with hypoglycemia $(<2.0 \mathrm{mmol} / \mathrm{l})$, Apgar score $<5$ after $5 \mathrm{~min}$, and the CT or TT 677 MTHFR genotype in the model. Hypothermia and sex were not found to be independent variables in this data set and were omitted from the final, reduced model.

From Table 5, the effects of low Apgar scores, hypoglycemia, and CT or TT MTHFR 677 genotype on the distribution of MRI patterns can be calculated. For example, in the presence of the CT or TT genotype, the log-odds for the WM/WS pattern increased by 1.74 (odds ratio $5.7 ; 95 \%$ confidence interval 1.8-17.8) and the same for the BGT pattern increased by 1.14 (odds ratio $3.1 ; 95 \%$ confidence interval 1.1-8.8).

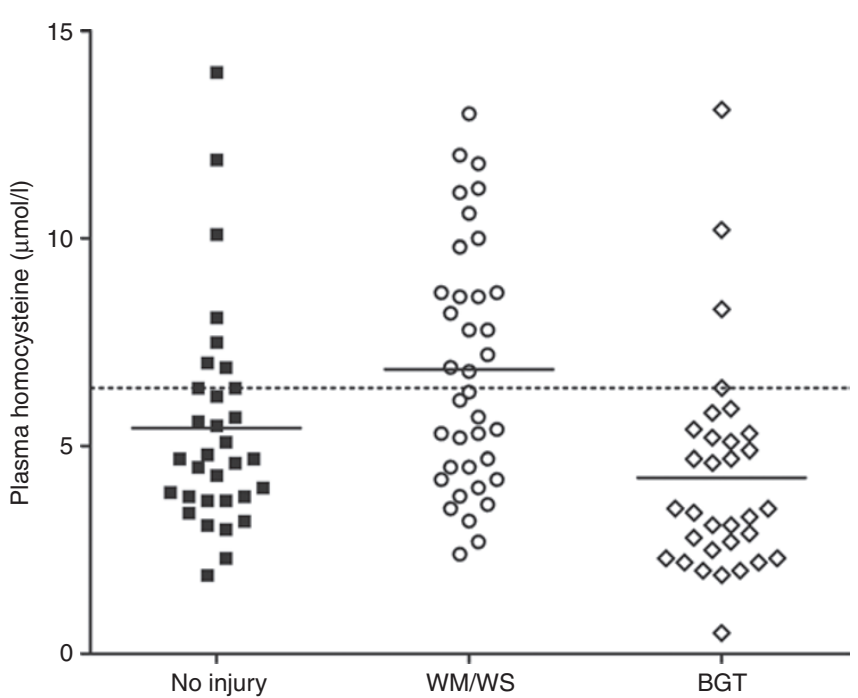

Figure 1. Homocysteine levels across the no injury, WM/WS, and BGT groups. The solid, short line indicates the median of the group. The dashed horizontal line indicates a tHcy level of $6.4 \mu \mathrm{mol} / \mathrm{l}$. Above this level is the upper quartile of the "no injury" group. BGT, basal ganglia-thalamus; tHcy, total homocysteine; WM/WS, white matter/watershed region.

Table 4. Mean homocysteine levels in plasma ( $\mu \mathrm{mol} / \mathrm{l})$ related to MTHFR genotype

\begin{tabular}{lcc}
\hline Normal & $\begin{array}{c}\text { 677 TT or 677 CT } \\
\text { genotype }\end{array}$ & $\begin{array}{c}\text { Other MTHFR } \\
\text { genotypes }\end{array}$ \\
\hline WM/WS & 5.23 & 5.51 \\
BGT & 6.58 & 6.81 \\
\hline
\end{tabular}

BGT, basal ganglia-thalamus; MTHFR, methylenetetrahydrofolate reductase;WM/WS, white matter/watershed region.

Table 5. Multinomial logistic regression analysis (coefficient (standard error))

\begin{tabular}{lcccc}
\hline & Intercept $^{\text {a }}$ & Hypoglycemia & $\begin{array}{c}\text { Apgar score } \\
<5(5 \mathrm{~min})\end{array}$ & $\begin{array}{c}\text { MTHFR 677 CT } \\
\text { orTT }\end{array}$ \\
\hline WM/WS & $0.098(.49)$ & $1.81(0.68)$ & $-1.74(0.58)$ & $1.74(0.57)$ \\
BGT & $-1.48(0.72)$ & $0.79(0.68)$ & $1.25(0.74)$ & $1.14(0.52)$
\end{tabular}

According to the model, the distribution of patients with no hypoglycemia $(<2.0 \mathrm{mmol} / \mathrm{l})$, an Apgar score $>5$ ( $5 \mathrm{~min})$, and no CT orTT MTHFR 677 genotype would result in $43 \%$ no injury, $47 \%$ WM/WS injury, and 10\% BGT injury. In patients with the CT or TT MTHFR 677 genotype, the percentages would be 12,79, and 9, respectively, according to the model. Likewise, distributions can be calculated for hypoglycemia or Apgar scores $<5$ (5 min) with or without the CT or TT 677 genotype being present.

BGT, basal ganglia-thalamus; MTHFR, methylenetetrahydrofolate reductase;WM/WS, white matter/watershed region.

anfants with no cerebral injury were the reference category.

\section{DISCUSSION}

This study examined the additional role of antenatal, perinatal, and thrombophilic factors in infants with NE and predominant WM/WS or BGT pattern of brain injury as compared with those with no injury on neonatal neuroimaging. Apgar scores $<5$ (5 min), hypoglycemia $<2.0 \mathrm{mmol} / \mathrm{l}$, and MTHFR $677 \mathrm{CT}$ or TT genotype were significant factors in the development of pattern of injury. tHcy in the upper quartile was significantly more often found in infants with WM/WS injury. 
Our results with respect to antenatal and postnatal clinical factors and the type of pattern of brain injury after NE are in agreement with previous findings (5-7). In the BGT group, Apgar scores were lower and emCS was more often required. All but one infant developed (sub)clinical seizures or had a flat background amplitude-integrated EEG (aEEG) pattern. The cord $\mathrm{pH}$ revealed a more severe acidosis in infants with BGT in contrast to those in the WM/WS group. This supports the association of profound, acute perinatal $\mathrm{HI}$ with predominant central gray matter damage.

Hypoglycemia $(<2.0 \mathrm{mmol} / \mathrm{l})$ was identified as an independent risk factor for WM/WS injury. Recently, Nadeem et al. (11) found that early hypoglycemia $(<6 \mathrm{~h})$ occurs frequently in infants with perinatal $\mathrm{HI}$ and appears to be related to the severity of $\mathrm{HI}$ and subsequent neurological outcome. This is consistent with the findings of Salhab et al. (12), who noted that hypoglycemia is an important risk factor for abnormal neurological outcome in term infants with fetal acidemia. Burns et al. (13) found white matter injury in $94 \%$ of infants with symptomatic hypoglycemia $(<2.6 \mathrm{mmol} / \mathrm{l})$ on their MRI performed during the first week after birth. In 7/28 infants with moderate/severe white matter injury, focal and small punctate lesions were present. From animal models, different pathogenic mechanisms of hypoglycemia in the onset of brain injury following $\mathrm{HI}$ have been described, such as the impairment of autoregulation (14). Yager et al. (15) found a marked reduction of brain infarctions in fasted rats with hypoglycemia and ketonemia as compared with insulin-treated and control rats that were subjected to HI. This might suggest that the presence of an alternative energy source in the absence of glucose is an important protection in perinatal $\mathrm{HI}$ brain damage.

Recent research has focused on mechanisms after HI, including excitotoxicity, oxidative stress, inflammation, and coagulation. Proinflammatory cytokines such as interleukin-1 $\beta$, interleukin-6, and tumor necrosis factor- $\alpha$ are significant contributors to $\mathrm{HI}$ brain injury $(8,16)$.

HI leads to a disturbed balance in hemostasis, predisposing to thrombosis (17). Adhami et al. (18) used the Levine/ Vannucci model in mice to mimic HI and showed that ischemia combined with hypoxemia results in local coagulation. They suggested that hypoxemia alters the balance between anti- and procoagulation properties of the endothelial cells in cerebral blood vessels. In addition, El Beshlawy et al. (19) found a marked decrease in the levels of the physiologic anticoagulants antithrombin, protein $\mathrm{C}$, and protein $\mathrm{S}$ predisposing to systemic thrombosis in infants with HI.

The current study focused on the additive role of thrombophilic factors, possibly underlying the vulnerabilities of brain injury after NE following HI. A role for thrombophilia has been described in different patterns of preterm and fullterm perinatal brain injury such as perinatal arterial ischemic stroke, intraventricular hemorrhage, and HI (20-22). To the best of our knowledge, thrombophilic abnormalities have not been studied previously in a prospective cohort of infants with $\mathrm{NE}$ in relation to subsequent patterns of brain injury. This study did not show a significant difference in the frequencies of factor V Leiden mutation, prothrombin gene mutation, or anticardiolipin antibodies across the three groups. However, the factor $\mathrm{V}$ Leiden mutation was present in only three infants with WM/WS injury. The point mutation in the factor V Leiden variant results in impaired degradation of activated factor $\mathrm{V}$ and, consequently, in hypercoagulability. The prothrombin gene mutation is associated with slightly increased plasma levels of prothrombin and a mild hypercoagulable state. Of note, there were differences in frequencies of the MTHFR polymorphisms and levels of tHcy (Tables 2, 3, and 5). Infants with WM/WS injury had the 677 CT or TT MTHFR genotype significantly more often, and higher levels of tHcy were found.

MTHFR converts 5,10-methylenetetrahydrofolate to 5-methyltetrahydrofolate, the major circulating form of folate. Folate is involved in the methylation of homocysteine. Mutations at positions -677 and -1298 of the MTHFR gene, especially the 677 TT variant, are associated with decreased activity of this enzyme, resulting in elevated tHcy (23). The prevalence of the TT genotype is $9 \%$ in the Dutch population (23). A mildly elevated tHcy has been linked to cardiovascular diseases and to cognitive dysfunction and neurological diseases (24). The causal effect of elevated tHcy might be explained by mechanisms such as neurotoxicity, impaired blood coagulation, and endothelial damage (24). Moreover, the MTHFR C677T variant was found to be a risk factor for congenital defects such as neural tube defects (25).

Dodelson de Kremer et al. (20) revealed an important role of the C677T allele in neonates with (unexplained) HI encephalopathy and subsequent severe multicystic encephalomalacia. Mothers of those infants all had hyperhomocysteinemia. A recent study in adults with atherosclerotic disease also showed that tHcy levels are associated with larger volumes of white matter lesions and an increased risk for lacunar infarcts (26). This suggests a role for tHcy in the association with cerebral small vessel diseases other than atherosclerosis. Endres et al. (27) found that folate deficiency and resultant hyperhomocysteinemia in mice were associated with increased oxidative DNA damage and ischemic lesion size after occlusion/reperfusion of the middle cerebral artery. However, the precise role of hyperhomocysteinemia and its relation with the C677T and A1298C MTHFR polymorphisms in neonatal brain damage has not been unraveled.

There was no association between MTHFR genotype and elevated tHcy, which is consistent with the findings in the neonates studied by Refsum et al. (28) (Table 4). Although folate deficiency is an important cause of hyperhomocysteinemia, Hogeveen et al. (29) demonstrated that administration of folinic acid in neonates did not lower tHcy concentrations. This could indicate that tHcy metabolism in the neonate may differ from metabolism later in life or that cobalamin might be a better strategy to lower tHcy levels. Molloy et al. (30) showed that maternal tHcy level is the primary predictor of tHcy in the fetus. Plasma folate exerts the highest influence on maternal tHcy levels. Consequently, to prevent high tHcy levels in the fetus or newborn infant, lowering maternal tHcy might be the key factor.

Elevated levels of $\mathrm{Lp}(\mathrm{a})$ have been considered to be prothrombotic because Lp(a) inhibits the binding of plasminogen to fibrinogen. In this study, none of the infants had an elevated 


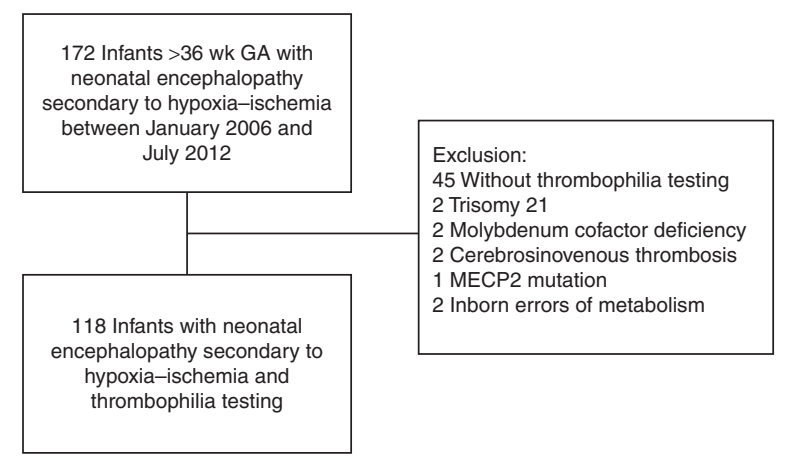

Figure 2. Flowchart of included patients. GA, gestational age.

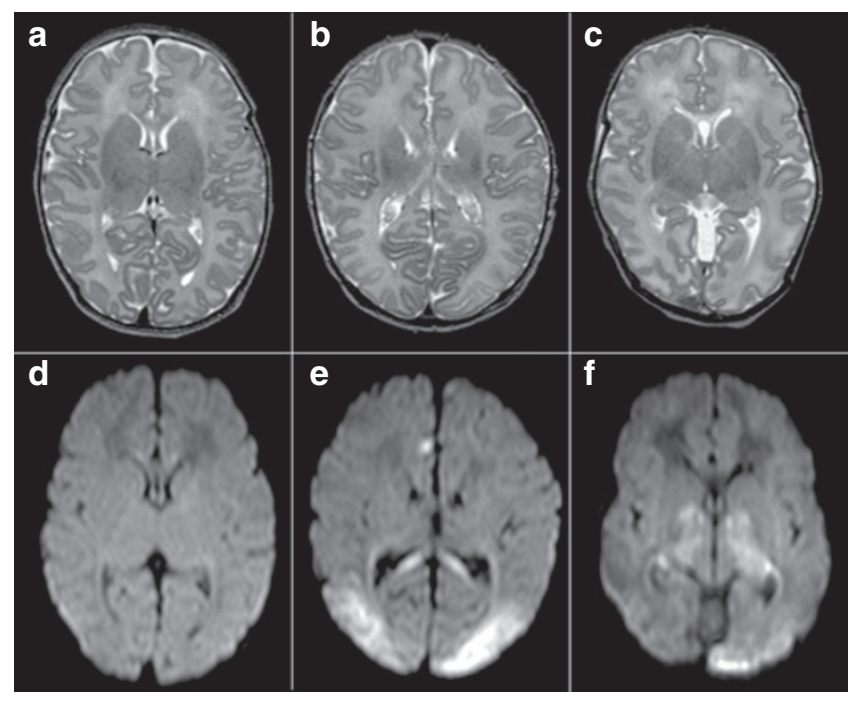

Figure 3. Magnetic resonance imaging (MRI) patterns after NE following HI. T2-weighted MRI (top row) with (a) normal pattern, (b) high signal intensity in the posterior WS region with partial loss of the cortical ribbon, and (c) mild increased signal intensity in the thalami. Bottom row shows the corresponding DW-MRI with (d) normal pattern and (e) bilateral posterior watershed and (f) BGT patterns of injury. BGT, basal ganglia-thalamus; DW, diffusion-weighted; $\mathrm{HI}$, hypoxia-ischemia; $\mathrm{NE}$, neonatal encephalopathy; WS, watershed.

level of $>300 \mathrm{mg} / \mathrm{dl}$, in contrast to a previous study in which elevated $\operatorname{Lp}(\mathrm{a})$ in infants with perinatal stroke was found as a common genetic risk factor (31).

The present study has several limitations. First, not all newborns underwent MRI because of either early death or absence of neonatal seizures in combination with a normal aEEG background pattern and normal sequential cUS findings. Although it is unlikely that infants in this latter group would have shown severe injury on an MRI, mild punctate white matter lesions may have been missed. Second, therapeutic hypothermia has been used from 2008 onward, whereas data were collected from 2006. Furthermore, some infants were referred outside the time window for therapeutic hypothermia or initially had only mild symptoms of NE. This might explain why hypothermia was not associated with injury pattern in the multinomial analysis. Third, mothers were not screened for thrombophilic factors. Previous studies have shown an association of thrombophilia in mothers with neonatal brain damage $(20,32)$. Fourth, levels of cobalamin, folate, and methionine were not measured in this cohort although they all significantly influence tHcy (28). Finally, blood for thrombophilia testing was taken at different points in time, although in $95 \%$ of the infants, this was within $8 \mathrm{~d}$ after birth. An association with tHcy and the day when blood was taken could not be established. Especially, tHcy and Lp(a) levels might be dependent on maternal levels. No levels of protein $C, S$, or antithrombin were examined, but these levels are known to be low in the first weeks after birth and within the range in which thrombotic disorders have been described in adults (33). The significance of low levels in neonatal thrombotic processes is still unknown.

Our results need to be confirmed in larger patient groups with NE after HI. If a low tHcy level proves critical to prevent (extensive) brain damage, prescription of folate and/or cobalamin has to be considered for mothers during the entire pregnancy.

In conclusion, infants with BGT predominant pattern of injury after NE due to HI showed lower Apgar scores, suggesting acute HI. However, hypoglycemia $(<2.0 \mathrm{mmol} / \mathrm{l})$, the presence of the MTHFR 677 CT or TT genotype, and higher levels of tHcy were independent variables associated with WM/WS injury. Ultimately, a better understanding of clinical and genetic factors that predispose to neuropathological substrates after HI followed by NE may lead to the unraveling of pathogenic mechanisms and identification of new intervention strategies.

\section{METHODS}

Infants with a diagnosis of NE and subsequent brain injury, born $>36$ wk gestational age, and admitted to the Level 3 neonatal intensive care unit of the Wilhelmina Children's Hospital in Utrecht, The Netherlands, between January 2006 and July 2012, were prospectively enrolled in this study. From January 2008, infants with NE but without significant brain injury on neuroimaging were also enrolled.

NE was defined as a clinical picture, occurring during the first $72 \mathrm{~h}$ after birth, consisting of one or more of the following symptoms: altered consciousness, irritability, abnormal tone pattern, convulsions, or lethargy. NE was considered to be secondary to perinatal asphyxia on the basis of at least two of the following criteria: (i) late decelerations on fetal monitoring or meconium-stained liquor, (ii) delayed onset of respiration, (iii) 5-min Apgar score $<7$, (iv) arterial umbilical cord $\mathrm{pH}<7.10$, and (v) multiorgan failure (4).

From January 2008, infants with a Thompson score $>7$, suggestive of moderate to severe NE, and admitted $<6 \mathrm{~h}$ after birth underwent total body cooling at $33-34^{\circ} \mathrm{C}$ for $72 \mathrm{~h}(34)$.

Of 127 infants with NE who had thrombophilic factors assessed, 118 infants were eligible for the study. Infants with suspected or confirmed congenital malformations, inborn errors of metabolism, or congenital infections were excluded (Figure 2).

No ethical permission was required by the medical ethical committee of our hospital for this retrospective, anonymous study.

\section{Neuroimaging}

Each infant underwent cUS on admission to document that there were no major developmental abnormalities. cUS was repeated daily during the first week to examine the evolution of lesions. Infants were subdivided into three groups: WM/WS or BGT predominant pattern of brain injury or no significant injury (Figure 3 ). On cUS, WM/WS injury was determined as echogenicity in the deep white matter and BGT injury as echogenicity of basal ganglia and thalami (35). MRI was performed in infants with ongoing or 
established abnormalities on cUS, in those who had clinical and/ or aEEG-confirmed neonatal seizures, and in those who received therapeutic hypothermia. MRI was performed using $1.5-\mathrm{T}$ and 3.0-T Philips system (Intera or Achieva Philips; Healthcare, Best, The Netherlands). MRI included sagittal T1-weighted, transverse T2-weighted, and inversion recovery-weighted sequences, as well as diffusion-weighted imaging.

On MRI, the WM/WS predominant pattern involves the spectrum of white matter injury, including watershed injury that affects the deep white matter and overlying cortex, and also lesions restricted to the periventricular white matter, the so-called punctate white matter lesions $(5,36)$. The BGT predominant pattern involves the deep gray nuclei and perirolandic cortex $(3,36,37)$. Infants with perinatal arterial ischemic stroke were not eligible for the study.

\section{Thrombophilia Investigations}

Blood samples were drawn from an indwelling arterialline. Investigations were performed according to a "thrombophilia protocol." Heterozygous or homozygous mutations of the factor V Leiden (G1691A), prothrombin (G20210A) gene, and C677T and A1298C polymorphisms in the MTHFR gene were performed by amplification and PCR analysis. Preceding factor $\mathrm{V}$ Leiden mutation analysis, activated protein $\mathrm{C}$ resistance was measured and levels $>0.90$ were considered as factor $\mathrm{V}$ Leiden-negative. The activated protein $\mathrm{C}$ resistance ratio was measured using a STA-Rack-Evolution (Diagnostica Stago, Asnieres sur Seine, France) in diluted factor V-deficient plasma to correct for coagulation factor deficiencies; this ratio was defined as the activated partial thromboplastin time in the presence of activated protein $\mathrm{C}$ divided by the activated partial thromboplastin time in the absence of activated protein C, corrected for the result of a normal pool.

Plasma Lp(a) and tHcy levels were measured using nefelometry (Siemens Behring BN-II, Marburg, Germany) and a chemiluminescence competitive immunoassay (Siemens Immulite, Erlangen, Germany), respectively. As previously defined in a study in children and infants, a plasma $\mathrm{Lp}$ (a) level of $>300 \mathrm{mg} / \mathrm{l}$ was set as a risk threshold value (38).

The levels of lupus anticoagulans were measured on a STA-Rack Evolution (Diagnostica Stago) using a diluted Russel Viper Venom time and an activated partial thromboplastin time according to the International Society on Thrombosis and Haemostasis recommendations $(39,40)$. Anticardiolipin and anti- $\beta-2$ glycoprotein- 1 immunoglobulin $\mathrm{G}$ and immunoglobulin $\mathrm{M}$ antibodies were analyzed using an enzyme-linked immunosorbent assay.

\section{Clinical Data}

Antepartum, perinatal, and postpartum risk factors were studied retrospectively. Antepartum factors included maternal fever $\left(>38^{\circ} \mathrm{C}\right)$ during delivery, preeclampsia defined as either preeclampsia or pregnancy-induced hypertension, and prolonged rupture of membranes $>24 \mathrm{~h}$. Perinatal factors were as follows: fetal bradycardia defined as late or variable decelerations or fetal distress, meconium-stained amniotic fluid, emCS, Apgar scores, umbilical artery $\mathrm{pH}$ or first arterial blood gas $<60 \mathrm{~min}$, birth weight, and head circumference. Multiorgan failure, hypglycemia $<2.0 \mathrm{mmol} / \mathrm{L}$ in the first 24 hrs after birth, and clinical or subclinical (aEEG-confirmed) seizures were noted as postnatal clinical factors.

\section{Statistical Analysis}

Infants were divided into three groups according to the predominant pattern of injury as described above. Clinical variables and thrombophilic factors were compared across the three patterns with $\chi^{2}$ tests for categorical variables and ANOVA or Kruskal-Wallis tests where appropriate for continuous variables. Statistical significance was defined as $P<0.05$. Analyses were performed using Predictive Analytics Software for Windows version 17.0 (SPSS, Chicago, IL).

The effect of the variables, which showed a significant difference in the univariable analysis on the distribution of WM/WS, BGT, or no brain injury, was assessed using a multinomial logistic regression model. Estimated coefficients from significant independent variables from the multinomial analysis were used to study the effects of the independent variables on the occurrence of WM/WS or BGT brain injury. For this multinomial analysis, "R"-software was used (version 2.14.0, The R Development Core Team, http:// cran.r-project.org/).

\section{STATEMENT OF FINANCIAL SUPPORT}

No financial assistance was received to support this study.

Disclosure: The authors declared no conflict of interest.

\section{REFERENCES}

1. Pierrat V, Haouari N, Liska A, Thomas D, Subtil D, Truffert P. Prevalence, causes, and outcome at 2 years of age of newborn encephalopathy: population based study. Arch Dis Child Fetal Neonatal Ed 2005;90:F257-61.

2. Ferriero DM. Neonatal brain injury. N Engl J Med 2004;351:1985-95.

3. de Vries LS, Groenendaal F. Patterns of neonatal hypoxic-ischaemic brain injury. Neuroradiology 2010;52:555-66.

4. Cowan F, Rutherford M, Groenendaal F, et al. Origin and timing of brain lesions in term infants with neonatal encephalopathy. Lancet 2003;361:736-42.

5. Li AM, Chau V, Poskitt KJ, et al. White matter injury in term newborns with neonatal encephalopathy. Pediatr Res 2009;65:85-9.

6. Mercuri E, Rutherford M, Barnett A, et al. MRI lesions and infants with neonatal encephalopathy. Is the Apgar score predictive? Neuropediatrics 2002;33:150-6.

7. Miller SP, Ramaswamy V, Michelson D, et al. Patterns of brain injury in term neonatal encephalopathy. J Pediatr 2005;146:453-60.

8. Leviton A, Dammann O. Coagulation, inflammation, and the risk of neonatal white matter damage. Pediatr Res 2004;55:541-5.

9. Calkavur S, Akisu M, Olukman O, et al. Genetic factors that influence short-term neurodevelopmental outcome in term hypoxic-ischaemic encephalopathic neonates. J Int Med Res 2011;39:1744-56.

10. Myers RE. Four patterns of perinatal brain damage and their conditions of occurrence in primates. Adv Neurol 1975;10:223-34.

11. Nadeem M, Murray DM, Boylan GB, Dempsey EM, Ryan CA. Early blood glucose profile and neurodevelopmental outcome at two years in neonatal hypoxic-ischaemic encephalopathy. BMC Pediatr 2011;11:10.

12. Salhab WA, Wyckoff MH, Laptook AR, Perlman JM. Initial hypoglycemia and neonatal brain injury in term infants with severe fetal acidemia. Pediatrics 2004;114:361-6.

13. Burns CM, Rutherford MA, Boardman JP, Cowan FM. Patterns of cerebral injury and neurodevelopmental outcomes after symptomatic neonatal hypoglycemia. Pediatrics 2008;122:65-74.

14. Anwar M, Vannucci RC. Autoradiographic determination of regional cerebral blood flow during hypoglycemia in newborn dogs. Pediatr Res 1988;24:41-5.

15. Yager JY, Heitjan DF, Towfighi J, Vannucci RC. Effect of insulin-induced and fasting hypoglycemia on perinatal hypoxic-ischemic brain damage. Pediatr Res 1992;31:138-42.

16. Hagberg H, Gilland E, Bona E, et al. Enhanced expression of interleukin (IL)-1 and IL-6 messenger RNA and bioactive protein after hypoxia-ischemia in neonatal rats. Pediatr Res 1996;40:603-9.

17. Bauman ME, Cheung PY, Massicotte MP. Hemostasis and platelet dysfunction in asphyxiated neonates. J Pediatr 2011;158(2 Suppl):e35-9.

18. Adhami F, Liao G, Morozov YM, et al. Cerebral ischemia-hypoxia induces intravascular coagulation and autophagy. Am J Pathol 2006;169:566-83.

19. El Beshlawy A, Hussein HA, Abou-Elew HH, Abdel Kader MS. Study of protein $\mathrm{C}$, protein $\mathrm{S}$, and antithrombin III in hypoxic newborns. Pediatr Crit Care Med 2004;5:163-6.

20. Dodelson de Kremer R, Grosso C. Maternal mutation $677 \mathrm{C}>\mathrm{T}$ in the methylenetetrahydrofolate reductase gene associated with severe brain injury in offspring. Clin Genet 2005;67:69-80.

21. Kenet G, Lütkhoff LK, Albisetti M, et al. Impact of thrombophilia on risk of arterial ischemic stroke or cerebral sinovenous thrombosis in neonates and children: a systematic review and meta-analysis of observational studies. Circulation 2010;121:1838-47.

22. Ramenghi LA, Fumagalli M, Groppo M, et al. Germinal matrix hemorrhage: intraventricular hemorrhage in very-low-birth-weight infants: 
the independent role of inherited thrombophilia. Stroke 2011;42: 1889-93.

23. Lievers KJ, Boers GH, Verhoef P, et al. A second common variant in the methylenetetrahydrofolate reductase (MTHFR) gene and its relationship to MTHFR enzyme activity, homocysteine, and cardiovascular disease risk. J Mol Med 2001;79:522-8.

24. Troen AM. The central nervous system in animal models of hyperhomocysteinemia. Prog Neuropsychopharmacol Biol Psychiatry 2005;29:1140-51.

25. van der Put NM, Blom HJ. Neural tube defects and a disturbed folate dependent homocysteine metabolism. Eur J Obstet Gynecol Reprod Biol 2000;92:57-61.

26. Kloppenborg RP, Nederkoorn PJ, van der Graaf Y, Geerlings MI. Homocysteine and cerebral small vessel disease in patients with symptomatic atherosclerotic disease. The SMART-MR study. Atherosclerosis 2011;216:461-6.

27. Endres M, Ahmadi M, Kruman I, Biniszkiewicz D, Meisel A, Gertz K. Folate deficiency increases postischemic brain injury. Stroke 2005;36:321-5.

28. Refsum H, Grindflek AW, Ueland PM, et al. Screening for serum total homocysteine in newborn children. Clin Chem 2004;50:1769-84.

29. Hogeveen M, den Heijer M, Schonbeck Y, et al. The effect of folinic acid supplementation on homocysteine concentrations in newborns. Eur J Clin Nutr 2010;64:1266-71.

30. Molloy AM, Mills JL, McPartlin J, Kirke PN, Scott JM, Daly S. Maternal and fetal plasma homocysteine concentrations at birth: the influence of folate, vitamin $\mathrm{B} 12$, and the 5,10-methylenetetrahydrofolate reductase 677C->T variant. Am J Obstet Gynecol 2002;186:499-503.

31. Günther G, Junker R, Sträter R, et al.; Childhood Stroke Study Group. Symptomatic ischemic stroke in full-term neonates: role of acquired and genetic prothrombotic risk factors. Stroke 2000;31:2437-41.
32. Simchen MJ, Goldstein G, Lubetsky A, Strauss T, Schiff E, Kenet G. Factor v Leiden and antiphospholipid antibodies in either mothers or infants increase the risk for perinatal arterial ischemic stroke. Stroke 2009;40:65-70.

33. Andrew M, Paes B, Milner R, et al. Development of the human coagulation system in the full-term infant. Blood 1987;70:165-72.

34. Thompson CM, Puterman AS, Linley LL, et al. The value of a scoring system for hypoxic ischaemic encephalopathy in predicting neurodevelopmental outcome. Acta Paediatr 1997;86:757-61.

35. Epelman M, Daneman A, Kellenberger CJ, et al. Neonatal encephalopathy: a prospective comparison of head US and MRI. Pediatr Radiol 2010;40:1640-50.

36. de Vries LS, Cowan FM. Evolving understanding of hypoxic-ischemic encephalopathy in the term infant. Semin Pediatr Neurol 2009;16:216-25.

37. Barkovich AJ, Miller SP, Bartha A, et al. MR imaging, MR spectroscopy, and diffusion tensor imaging of sequential studies in neonates with encephalopathy. AJNR Am J Neuroradiol 2006;27:533-47.

38. Nowak-Göttl U, Junker R, Hartmeier M, et al. Increased lipoprotein(a) is an important risk factor for venous thromboembolism in childhood. Circulation 1999;100:743-8.

39. Brandt JT, Triplett DA, Alving B, Scharrer I. Criteria for the diagnosis of lupus anticoagulants: an update. On behalf of the Subcommittee on Lupus Anticoagulant/Antiphospholipid Antibody of the Scientific and Standardisation Committee of the ISTH. Thromb Haemost 1995;74:1185-90.

40. Pengo V, Tripodi A, Reber G, et al. Update of the guidelines for lupus anticoagulant detection. Subcommittee on Lupus Anticoagulant/Antiphospholipid Antibody of the Scientific and Standardisation Committee of the International Society on Thrombosis and Haemostasis. J Thromb Haemost 2009;7:1737-40. 